\title{
SUR UNE NOUVELLE CLASSE DE SURFACES.
}

\author{
Par M. Georges Tzitzéica (Bucarest).
}

Adunanza del ro novembre igo7.

L'étude de la déformation des surfaces tétraédrales

$$
A x^{\frac{2}{3}}+B y^{\frac{2}{3}}+C z^{\frac{2}{3}}=\mathrm{I}
$$

conduit à la recherche des surfaces pour lesquelles la courbure totale est proportionnelle à la quatrième puissance de la distance d'un point fixe au plan tangent.

Je désignerai par $S$ toute surface qui jouit de la propriété précédente et par centre de $S$ le point fixe qui figure dans la définition de cette surface.

I. Supposons donnée une surface $S$ à courbure totale négative. On pourra prendre, sans restreindre la généralité,

$$
R^{\prime} R^{\prime \prime}=-\frac{\mathrm{I}}{p^{4}}
$$

où $R^{\prime}$ et $R^{\prime \prime \prime}$ sont les rayons principaux de courbure et $p$ la distance du centre de $S$ (qui sera pris pour origine) au plan tangent.

Prenons comme coordonnées curvilignes sur $S$ ses lignes asymptotiques. Les formules de M. Lelieuvre (Darboux, IV partie, page 24; Btanchi, Volume I, p. I62) donnent, pour un point $M(x, y, z)$ de la surface,

$$
\frac{\partial x}{\partial u}=y_{1} \frac{\partial z_{1}}{\partial u}-z_{1} \frac{\partial y_{1}}{\partial u}, \quad \frac{\partial x}{\partial v}=-\left(y_{1} \frac{\partial z_{1}}{\partial v}-z_{1} \frac{\partial y_{1}}{\partial v}\right)
$$

et des formules analogues pour $y$ et $z ; x_{1}, y_{1}, z_{1}$ sont des intégrales d'une équation de la forme

(3)

Or, on a

et, comme

on obtient de ( 1 )

$$
\frac{\partial^{2} \theta}{\partial u \partial v}=k \theta \text {. }
$$

$$
x_{1}^{2}+y_{1}^{2}+z_{I}^{2}=\sqrt{-R^{\prime} R^{\prime \prime}},
$$

$$
p=\frac{x_{1} x+y_{1} y+z_{1} z}{\sqrt{x_{1}^{2}+y_{1}^{2}+z_{1}^{2}}},
$$

$$
x_{1} x+y_{1} y+z_{1} z= \pm I \text {. }
$$


En changeant au besoin les signes de $x_{1}, y_{\mathrm{I}}, z_{\mathrm{I}}$, on pourra prendre

$$
x_{1} x+y_{\mathrm{r}} y+z_{\mathrm{r}} z=\mathrm{I} \text {. }
$$

On peut donc énoncer le résultat suivant: Toute surface définie par les formules (2), $x_{\mathrm{r}}, y_{1}, z_{1}$ étant trois intégrales d'une équation de la forme (3) et vérifiant la relation (4), est une surface $S$.

2. On tire aisément des égalités (2) les relations suivantes

$$
\begin{aligned}
& \int \sum x_{\mathrm{s}} \frac{\partial x}{\partial u}=0, \quad \sum x_{\mathrm{r}} \frac{\partial x}{\partial v}=0, \\
& \sum \frac{\partial x_{I}}{\partial u} \frac{\partial x}{\partial u}=0, \quad \sum \frac{\partial x_{I}}{\partial v} \frac{\partial x}{\partial v}=0 .
\end{aligned}
$$

D'autre part, en dérivant (4) et en tenant compte des relations précédentes, on obtient

$$
\sum x \frac{\partial x_{1}}{\partial u}=0, \quad \sum x \frac{\partial x_{1}}{\partial v}=0 .
$$

Considérons maintenant la surface $S_{1}$ décrite par le point $M_{1}\left(x_{1}, y_{1}, z_{1}\right)$. On voit sur les rélations ( 5 ) et (6) que la normale au point $M(x, y, z)$ de $S$ est parallèle d $O M_{\text {t }}$ et que la normale en $M_{\mathrm{r}}$ à $S_{\mathrm{r}}$ est parallèle a $O M$.

Prenons de $(5)$ et $(6)$ les relations

$$
\sum x \frac{\partial x_{1}}{\partial u}=0, \quad \sum \frac{\partial x}{\partial u} \frac{\partial x_{1}}{\partial u}=0 ;
$$

on en tire immédiatement

$$
\frac{\partial x_{1}}{\partial u}=\lambda\left(z \frac{\partial y}{\partial u}-y \frac{\partial z}{\partial u}\right)
$$

et des égalités analogues pour $y_{1}$ et $z_{\mathrm{r}}$. Or, remplaçons $\frac{\partial y}{\partial u}$ et $\frac{\partial z}{\partial u}$ par leurs valeurs tirées de (2); ọn obtient

$$
\begin{aligned}
\frac{\partial x_{1}}{\partial u} & =\lambda\left[z\left(z_{1} \frac{\partial x_{1}}{\partial u}-x_{1} \frac{\partial z_{1}}{\partial u}\right)-y\left(x_{1} \frac{\partial y_{1}}{\partial u}-y_{1} \frac{\partial x_{1}}{\partial u}\right)\right] \\
& =\lambda\left[\frac{\partial x_{1}}{\partial u}\left(x x_{1}+y y_{1}+z z_{1}\right)-x_{1}\left(x \frac{\partial x_{1}}{\partial u}+y \frac{\partial y_{1}}{\partial u}+z \frac{\partial z_{I}}{\partial u}\right)\right] \\
& =\lambda \frac{\partial x_{1}}{\partial u},
\end{aligned}
$$

d'où $\lambda=\mathrm{I}$ et par conséquent

$$
\frac{\partial x_{t}}{\partial u}=z \frac{\partial y}{\partial u}-y \frac{\partial z}{\partial u}
$$

de la même manière on trouve

$$
\frac{\partial x_{1}}{\partial v}=-\left(z \frac{\partial y}{\partial v}-y \frac{\partial z}{\partial v}\right)
$$

En égalant les deux valeurs de $\frac{\partial^{2} x_{x}}{\partial u \partial v}$ tirées des deux dernières égalités, et en faisant la même chose pour $\frac{\partial^{2} y_{x}}{\partial u \partial v}$ et $\frac{\partial^{2} z_{\mathrm{t}}}{\partial u \partial v}$, on trouve que $x, y, z$ sont trois intégrales 
d'une equation de la forme

(7)

$$
\frac{\partial^{2} \omega}{\partial u \partial v}=b \omega
$$

Or, le théoreme établi à la fin du $\int_{\text {I }}$ prouve alors que $S_{1}$ est une surface $S$.

De plus, comme il est facile de voir que $S_{t}$ est la polaire réciproque de la surface $S$ décrite par $M(x, y, z)$, par rapport à la sphère de rayon I ayant son centre d l'origine, on a le résultat suivant: Étant donnée une surface $S$ de centre $O$, sa polaire réciproque par rapport à une spbère de centre $O$ est aussi une surface $S$.

Il résulte encore des formules précédentes pour $\frac{\partial x_{1}}{\partial u}$ et $\frac{\partial x_{1}}{\partial v}$ que les lignes $u=$ const. et $v=$ const. sont les lignes asymptotiques de $S_{1}$, ce qui est d'ailleurs conforme dan théorème connu.

3. Comme la surface $S$ décrite par le point $M(x, y, z)$ est rapportée à ses lignes asymptotiques, $x, y, z$ vérifient, en dehors de (7), deux équations linéaires de la forme

$$
\left\{\begin{array}{l}
\frac{\partial^{2} \omega}{\partial u^{2}}=a \frac{\partial \omega}{\partial u}+b \frac{\partial \omega}{\partial v}, \\
\frac{\partial^{2} \omega}{\partial v^{2}}=a^{\prime} \frac{\partial \omega}{\partial u}+b^{\prime} \frac{\partial \omega}{\partial v} .
\end{array}\right.
$$

Le système (7) et (8) admettant trois intégrales linéairement indépendantes, on a les conditions suivantes

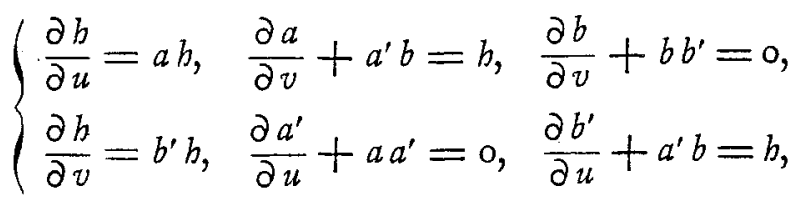

que nous étudierons plus loin.

On a par conséquent le résultat suivant:

Les coordonnées cartésiennes d'une surface $S$ (le centre de cette surface étant pris pour origine) vérifient un système d'équations linéaires aux dérivées partielles de la forme des équations (7) et (8).

On a de même pour la surface $S_{1}$, d'abord l'équation (3) et puis deux autres équations

$$
\left\{\begin{array}{l}
\frac{\partial^{2} \theta}{\partial u^{2}}=a_{\mathrm{r}} \frac{\partial \theta}{\partial u}+b_{\mathrm{r}} \frac{\partial \theta}{\partial v} \\
\frac{\partial^{2} \theta}{\partial v^{2}}=a_{1}^{\prime} \frac{\partial \theta}{\partial u}+b_{1}^{\prime} \frac{\partial \theta}{\partial v}
\end{array}\right.
$$

$a_{1}, b_{1}, a_{1}^{\prime}, b_{1}^{\prime}$ et $k$ étant liés par des relations toutes pareilles à (9). Remarquons que, a l'aide des expressions de $\frac{\partial x_{1}}{\partial u}$ et $\frac{\partial x_{1}}{\partial v}$ trouvées plus haut, on peut facilement obtenir $\frac{\partial^{2} x_{1}}{\partial u^{2}}$ et $\frac{\partial^{2} x_{I}}{\partial u^{2}}$. On en déduit

et de ld $k=h$.

$$
a_{\mathrm{r}}=a, \quad b_{\mathrm{s}}=-b, \quad a_{\mathrm{r}}^{\prime}=-a^{\prime}, \quad b_{\mathrm{t}}^{\prime}=b^{\prime}
$$


On voit donc le changement qui se fait dans le système des équations (7) et (8), lorsque l'on passe de la surface $S$ d̀ sa polaire réciproque $S_{1}$.

4. Je me propose de démontrer maintenant que si $x, y, z$ sont trois solutions linéairement indépendantes d'un systéme de la forme des équations (7) et (8), le point $M(x, y, z)$ décrit une surface $S$ ayant l'origine pour centre.

En effet, comme $x, y, z$ sont des intégrales de l'équation (7), les formules

$$
\frac{\partial x_{x}}{\partial u}=z \frac{\partial y}{\partial u}-y \frac{\partial z}{\partial u}, \quad \frac{\partial x_{x}}{\partial v}=-\left(z \frac{\partial y}{\partial v}-y \frac{\partial z}{\partial v}\right)
$$

et les analogues en $y_{t}$ et $z_{1}$, définissent d'après le théorème de M. Lelieuvre une surface $S_{x}$ rapportée à ses lignes asymptotiques.

Il résulte de là que $x_{1}, y_{1}, z_{1}$ sont des intégrales d'un système de la forme ( $\left.8^{\prime}\right)$.

Posons maintenant

$$
\omega^{\prime}=x x_{1}+y y_{1}+z z_{1} .
$$

On a, en tenant compte de (10),

$$
\frac{\partial \omega^{\prime}}{\partial u}=\sum x_{i} \frac{\partial x}{\partial u}, \quad \frac{\partial \omega^{\prime}}{\partial v}=\sum x_{i} \frac{\partial x}{\partial v}
$$

et en vertu de (7) et (8)

et

$$
\left\{\begin{array}{l}
\frac{\partial^{2} \omega^{\prime}}{\partial u^{2}}=a \frac{\partial \omega^{\prime}}{\partial u}+b \frac{\partial \omega^{\prime}}{\partial v} \\
\frac{\partial^{2} \omega^{\prime}}{\partial v^{2}}=a^{\prime} \frac{\partial \omega^{\prime}}{\partial u}+b^{\prime} \frac{\partial \omega^{\prime}}{\partial v}
\end{array}\right.
$$

ou่

$$
\frac{\partial^{2} \omega^{\prime}}{\partial u \partial v}=b \omega^{\prime}+M
$$

$$
M=\sum \frac{\partial x_{i}}{\partial v} \frac{\partial x}{\partial u}=\sum \frac{\partial x}{\partial u} \frac{\partial x}{\partial v}
$$

Or, on tire de ces expressions de $M$

$$
\frac{\partial M}{\partial u}=a M, \quad \frac{\partial M}{\partial v}=b^{\prime} M
$$

et, en remarquant les relations

$$
\frac{\partial b}{\partial u}=a b, \quad \frac{\partial b}{\partial v}=b^{\prime} b
$$

tirées de (9), on a $M=-m b, m=$ constante. En remplaçant en ( $\left.7^{\prime}\right)$, on obtient

$$
\frac{\partial^{2}\left(\omega^{\prime}-m\right)}{\partial u \partial v}=b\left(\omega^{\prime}-m\right) \text {, }
$$

ce qui prouve que $\omega^{\prime}-m$ est une intégrale du système formé par les équations (7) et (8). Comme ce système n'a que trois intégrales linéairement indépendantes $x, y$ et $z$ on a

$$
\omega^{\prime}-m=x x+\beta y+\gamma z
$$


$\alpha, \beta$ et $\gamma$ étant des constantes, ou

$$
x\left(x_{i}-x\right)+y\left(y_{1}-\beta\right)+z\left(z_{i}-\gamma\right)=m .
$$

D'après les formules (ro) on voit que l'on peut ajouter $d x_{1}, y_{1}, z_{x}$ des constantes arbitraires; on peut donc ecrire

$$
x x_{1}+y y_{1}+z z_{1}=m \text {. }
$$

Il résulte de là, que la surface $S_{1}$ est une surface $S$, et par conséquent sa polaire réciproque, la surface décrite par le point $M(x, y, z)$, est aussi une surface $S$.

C'est là le théorème fondamental que je voulais établir.

5. Il est clair que si $x, y, z$ sont trois intégrales, linéairement indépendantes, des équations (7) et (8), il en est de même de

$$
\left\{\begin{array}{l}
x^{\prime}=a_{1} x+b_{1} y+c_{1} z \\
y^{\prime}=a_{2} x+b_{2} y+c_{2} z, \\
z^{\prime}=a_{3} x+b_{3} y+c_{3} z
\end{array}\right.
$$

les $a, b, c$ étant des constantes dont le déterminant est différent de zéro. Le point $M^{\prime}\left(x^{\prime}, y^{\prime}, z^{\prime}\right)$ décrit par conséquent aussi une surface $S$.

Donc, toute transformation linéaire de la forme (II) change une surface $S$ en une autre surface $S$ :

En combinant cette transformation avec une autre trouvece plus haut, on a: Une transformation par polaires réciproques par rapport dे une quadrique d̀ centre ayant son centre au centre d'une surface $S$, transforme cette surface $S$ en une autre surface $S$.

6. Je me propose maintenant de trouver l'origine des propriétés précédentes des surfaces $S$ par rapport $\downarrow$ une transformation linéaire de la forme (II) et d une transformation par polaires réciproques.

Je vais montrer d'abord qu'une transformation de la forme (II) ne change pas le rapport éntre la courbure totale et la quatrième puissance de la distance de l'origine au plan tangent d'une surface. On démontre ce théorème aisément à l'aide de l'expression suivante

$$
K=-\frac{I}{\left[\left(\frac{\partial f}{\partial x}\right)^{2}+\left(\frac{\partial f}{\partial y}\right)^{2}+\left(\frac{\partial f}{\partial z}\right)^{2}\right]^{2}} \cdot\left|\begin{array}{cccc}
\frac{\partial^{2} f}{\partial x^{2}} & \frac{\partial^{2} f}{\partial x \partial y} & \frac{\partial^{2} f}{\partial x \partial z} & \frac{\partial f}{\partial x} \\
\frac{\partial^{2} f}{\partial y \partial x} & \frac{\partial^{2} f}{\partial y^{2}} & \frac{\partial^{2} f}{\partial y \partial z} & \frac{\partial f}{\partial y} \\
\frac{\partial^{2} f}{\partial z \partial x} & \frac{\partial^{2} f}{\partial z \partial y} & \frac{\partial^{2} f}{\partial z^{2}} & \frac{\partial f}{\partial z} \\
\frac{\partial f}{\partial x} & \frac{\partial f}{\partial y} & \frac{\partial f}{\partial z} & 0
\end{array}\right|
$$

pour la courbure totale et où $f(x, y, z)=0$ est l'équation de la surface. En employant les coordonnées homogènes le théorème est presque évident.

Passons $₫$ la transformation par polaires réciproques par rapport d une sphère de rayon un. Je vais demontrer que cette transformation change le rapport entre la courbure totale et la quatrième puissance de la distance de l'origine au plan tangent, en son inverse. 
Soient $S$ et $S^{\prime}$ les deux surfaces polaires réciproques par rapport à la sphère $\mathbf{\Sigma}$ de rayon un et de centre o; $d s$ et $d s^{\prime}$ les éléments d'aire autour de deux points cortespondants $M$ et $M^{\prime}$; $d$ o et $d \sigma^{\prime}$ les éléments d'aire de leurs représentations sphériques sur $\Sigma ; K$ et $K^{\prime}$ leurs courbures totales en $M$ et $M^{\prime}$. Posons enfin $\lambda=\cos M O M^{\prime}$. On a alors

Or

$$
K=\frac{d \sigma}{d s}, \quad K^{\prime}=\frac{d \sigma^{\prime}}{d s^{\prime}}, \quad \frac{\lambda d s^{\prime}}{d \sigma}={\overline{O M^{\prime}}}^{2}, \quad \frac{\lambda d s}{d \sigma^{\prime}}=\overline{O M}^{2} .
$$

donc

$$
O M=\frac{\mathrm{I}}{p^{\prime}}, \quad O M^{\prime}=\frac{\mathrm{r}}{p}, \quad \lambda=p p^{\prime}
$$

$$
\frac{K}{p^{4}} \cdot \frac{K^{\prime}}{p^{\prime 4}}=\mathrm{x}
$$

7. Revenons maintenant au système (7) et (8) et aux relations (9). Il faut remarquer tout d'abord, que l'on ne peut pas avoir $b=0$; autrement, si $x, y$, $z$ sont trois intégrales linéairement indépendantes du système, comme $m=$ const. est aussi, dans ce cas, une intégrale, on a

$$
m=\alpha x+\beta y+\gamma z
$$

ce qui prouve que la surface est un plan.

On a donc $h \neq 0$, et alors le systeme (9) donne

$$
\left\{\begin{array}{cc}
a=\frac{\mathrm{I}}{b} \frac{\partial b}{\partial u}, & b=\frac{U}{b}, \quad a^{\prime}=\frac{V}{b}, \quad b^{\prime}=\frac{\mathrm{I}}{b} \frac{\partial b}{\partial v}, \\
\frac{\partial^{2} \log b}{\partial u \partial v}=b-\frac{U V}{b^{2}} .
\end{array}\right.
$$

Prenons d'abord $U=0, V=0$; on a

d'où

$$
\frac{\partial^{2} \log b}{\partial u \partial v}=h
$$

$$
b=-\frac{2 U_{\mathrm{I}}^{\prime} V_{\mathrm{x}}^{\prime}}{\left(\mathrm{I}+U_{\mathrm{r}} V_{\mathrm{I}}\right)^{2}} \text {. }
$$

On déduit de là les valeurs de $a$ et $b^{\prime}$ et en les introduisant dans le système (7) et (8) et en prenant pour variables indépendantes $\alpha=U_{1}, \beta=V_{1}$, on obtient le système

$$
\frac{\partial^{2} \omega}{\partial \alpha^{2}}+\frac{2 \beta}{\mathrm{I}+\alpha \beta} \frac{\partial \omega}{\partial \alpha}=0, \quad \frac{\partial^{2} \omega}{\partial \beta^{2}}+\frac{2 \alpha}{\mathrm{I}+\alpha \beta} \frac{\partial \omega}{\partial \beta}=0, \quad \frac{\partial^{2} \omega}{\partial \alpha \partial \beta}=-\frac{2 \omega}{(\mathrm{I}+\alpha \beta)^{2}} .
$$

On a immédiatement trois intégrales linéairement indépendantes

$$
x=\frac{\alpha+\beta}{I+\alpha \beta}, \quad y=i \frac{\beta-\alpha}{I+\alpha \beta}, \quad z=\frac{\alpha \beta-I}{I+\alpha \beta} \text { (DarboUx, IV, p. 97). }
$$

La surface $S$ correspondante est une sphère. A l'aide d'une transformation (II) on déduit que les quadriques à centre sont aussi des surfaces $S$.

8. Prenons maintenant dans les formules (12) $V=0, U \neq 0$. On réduit alors 
le systeme $(7)$ et $(8)$ au suivant

$$
\begin{aligned}
\frac{\partial^{2} \omega}{\partial \alpha^{2}}+\frac{2 \beta}{I+\alpha \beta} \frac{\partial \omega}{\partial \alpha} & =-(I+\alpha \beta)^{2} A \frac{\partial \omega}{\partial \beta} \quad(A=\text { fonct. de } \alpha), \\
\frac{\partial^{2} \omega}{\partial \beta^{2}}+\frac{2 \alpha}{-+\alpha \beta} \frac{\partial \omega}{\partial \beta} & =0, \\
\frac{\partial^{2} \omega}{\partial \alpha \partial \beta} & =-\frac{2 \omega}{(I+\alpha \beta)^{2}} .
\end{aligned}
$$

Il n'est pas difficile de démontrer que le système admet les trois intégrales linéairement indépendantes

$$
\omega_{i}=\frac{2 \beta}{\mathrm{I}+\alpha \beta} \cdot \varphi_{i}(x)-\varphi_{i}^{\prime}(\alpha) \quad(i=\mathrm{I}, 2,3),
$$

$\varphi_{1}, \varphi_{2}$ et $\varphi_{3}$ étant trois intégrales linéairement indépendantes de l'équation

$$
\varphi^{\prime \prime \prime}(\alpha)-2 A \varphi(\alpha)=0 \text {. }
$$

On a de cette manière un nombre illimité de surfaces $S$ dépendant de la fonction arbitraire $A$ de $\alpha$.

Si l'on prend, par exemple, $A=\frac{1}{2}$, on trouve la surface

$$
(y z+z x+x y)^{3}=2 \zeta(x y z+1)^{2} .
$$

Cette surface, comme celles où $A$ est une fonction arbitraire de $\alpha$, est une surface réglée. Il est aisé de voir que l'on a obtenu de cette manière toutes les surfaces $S$ réglées.

9. Supposons maintenant $U \neq 0, V \neq 0$. En remplaçant $b$ par $b_{\mathrm{s}}=b \sqrt[3]{U V}$, et en faisant le changement de variables $\alpha=\int \sqrt[3]{U} d u, \beta=\int \sqrt[3]{V} d v$ on obtient le système

$$
\left\{\begin{aligned}
\frac{\partial^{2} \omega}{\partial \alpha^{2}} & =\frac{\mathrm{I}}{b} \frac{\partial b}{\partial \alpha} \frac{\partial \omega}{\partial \alpha}+\frac{\mathrm{I}}{b} \frac{\partial \omega}{\partial \beta} \\
\frac{\partial^{2} \omega}{\partial \beta^{2}} & =\frac{\mathrm{I}}{b} \frac{\partial \omega}{\partial \alpha}+\frac{\mathrm{I}}{b} \frac{\partial b}{\partial \beta} \frac{\partial \omega}{\partial \beta} \\
\frac{\partial^{2} \omega}{\partial \alpha \partial \beta} & =b \omega
\end{aligned}\right.
$$

où $b$ est une intégrale de l'équation

$$
\frac{\partial^{2} \log b}{\partial \alpha \partial \beta}=b-\frac{I}{b^{2}} .
$$

On a une intégrale immédiate de (14), $b=m$, où $m^{j}=\mathbf{I}$.

Le système (13) s'intègre alors facilement, et on obtient les trois intégrales

$$
x=e^{m_{\mathrm{I}}\left(\alpha+m m_{\mathrm{1}} \beta\right)}, \quad y=e^{m_{2}\left(\alpha+m m_{2} \beta\right)}, \quad z=e^{m_{3}\left(\alpha+m m_{3} \beta\right)} \quad\left(m_{i}^{3}=1, i=1,2,3\right),
$$

d'oú l'équation de la surface

$$
\log x+\log y+\log z=0
$$


ou

$$
x y z=\mathrm{I}
$$

et à l'aide de la transformation (II)

$$
\left(a_{1} x+b_{1} y+c_{1} z\right)\left(a_{2} x+b_{2} y+c_{2} z\right)\left(a_{3} x+b_{3} y+c_{3} z\right)=1 .
$$

Parmi ces surfaces $S$ il y en a une de révolution

$$
z\left(x^{2}+y^{2}\right)=\mathrm{I} \text {. }
$$

En définitive, pour avoir toutes les surfaces $S$ non-réglées il faut intégrer d'abord l'équation (14) et ensuite le système correspondant (13).

Bucarest, 30 octobre 1907.

G. TZITZÉiCA. 